\title{
summary
}

\section{Patients report more discomfort after surgical endodontic retreatment than non-surgical}

Kvist T, Reit C. Postoperative discomfort associated with surgical and nonsurgical endodontic retreatment. Endod Dent Traumatol 2000; 16:71-74

Objective To observe any systematic difference between surgical and non-surgical endodontic retreatment of endodontic failures.

Design Randomised controlled trial.

Intervention Surgical or non-surgical endodontic retreatment.

Outcome measures Patients recorded pain and swelling each postoperative day for 1 week using a visual analogue scale (VAS). Use of analgesia was also recorded.

Results Ninety-two patients were included in the study. Pain and swelling was significantly higher in the surgically retreated patients with $67 \%$ of patients in the surgical group recording use of analgesics compared with $16 \%$ in the non-surgical group. Only surgical patients reported absences from work post-treatment.

Conclusion Surgical retreatment resulted in more pain and swelling than non-surgical retreatment.

Address for reprints: Thomas Kvist, Gothenburg University, Institute of Odontology, PO Box 450, SE 40530 Gothenburg, Sweden.

\section{Commentary}

This trial was designed to compare the incidence of postoperative pain, swelling and subsequent absence from work as a result of non-surgical or surgical retreatment of a previously root-canal-treated-tooth which failed to heal. Using the same population of patients included in a previous study, ${ }^{1} 92$ patients with 95 previously treated maxillary and mandibular anterior teeth were randomly selected using the 'minimization method' for non-surgical or surgical retreatment. For 7 days following either retreatment modality, the patients assessed their own respective postoperative swelling and pain using a VAS which ranged from "no swelling" to "very severe swelling" and "no pain" to "intolerable pain". The patients were also asked to record whether they required analgesics for pain relief and if they had to take any time off from work as a result of the pain and/or swelling. It should be noted that the patients were not prescribed any medication postoperatively, nor instructed to use cold compresses postsurgically. Thus, if needed, over-the-counter analgesics had to be used. The results were analysed using a chi-square test with Yates' correction and Wilcoxon's rank sum test.

The authors reported an excellent return of 93 questionnaires. The results indicated that the patients in the surgical retreatment group experienced significantly more postoperative pain and swelling than those in the nonsurgical retreatment group. In addition, more patients in the surgical group required analgesics to control their pain, and absence from work was only reported by patients in the surgical group and the main reasons for the absence were swelling and discoloration, not pain.

Although the results of this study indicated that there is more postoperative discomfort, swelling, absence from work and increased indirect costs to the patient after having surgical retreatment of an endodontically treated tooth which has failed to heal, there is no indication that nonsurgical retreatment is the preferred treatment. What it does indicate is that practitioners should inform their patients that they may experience more postoperative pain and swelling and that they should consider prescribing a non-steroidal anti-inflammatory drug post-treatment and instructing their patients in the use of cold compresses.

In pain studies such as this one, it is always difficult to control variables such as the complexity and length of the surgical procedure, individual differences in pain tolerance, and the fact that patients have a preconceived notion that surgery results in increased pain and swelling. Although the authors attempted to control these variables, they are inherent in this type of clinical trial and the patients could not be blinded from the procedure that had been performed.

1. Kvist $\mathrm{T}$, Reit $\mathrm{C}$. Results of endodontic retreatment: a randomised clinical study comparing surgical and non-surgical procedures. J Endod 1999; 25:814-817.

Jeffrey W Hutter
Department of Endodontics
Goldman School of
Dental Medicine,
Boston University, Boston,
Massachusetts,
USA

